\title{
Supraceliac aortic cross-clamping to control bleeding from the celiac axis during pancreatic surgery: a case report
}

Yuki Takano ${ }^{1 \dagger}$, Shuichi Fujioka ${ }^{1 *+} \circledast$ , Hironori Shozaki ${ }^{1 \dagger}$, Naoki Toya ${ }^{1}$ and Toru Ikegami ${ }^{2}$

\begin{abstract}
Background: Intraoperative bleeding from the celiac axis (CA) can occur during pancreatic surgery, and appropriate management is essential to avoid critical complications. Here, we have reported a case that was managed with supraceliac aortic cross-clamping (SAC) for arterial bleeding from the CA during pancreatic surgery.

Case presentation: A 70-year-old man was diagnosed with pancreatic cancer located in the pancreatic head and body. Preoperative computed tomography showed a stricture at the root of the CA, which may have been caused by a median arcuate ligament. Pancreaticoduodenectomy with division of the median arcuate ligament was scheduled. Uncontrollable bleeding from the root of the CA was observed during surgery. The bleeding was controlled by performing SAC, and a defect in the CA was confirmed. Arterial wall repair was successfully performed under temporal blood control using SAC. The aortic clamp time was $2 \mathrm{~min}$ and $51 \mathrm{~s}$, and the intraoperative blood loss was $480 \mathrm{ml}$.

Conclusions: Although SAC is primarily a procedure for ruptured abdominal aortic aneurysm, it can be useful for the management of CA injuries during pancreatic surgery.
\end{abstract}

Keywords: Intraoperative injury, Celiac axis, Arterial clamping, Pancreatic surgery

\section{Background}

The celiac axis (CA) is a major artery that is managed during pancreatic surgery, especially surgery for pancreatic cancer. Recently, distal pancreatectomy with CA resection has become a standard option for locally advanced pancreatic body/tail cancer with the development of neoadjuvant chemotherapy [1-3]. Therefore, intraoperative injury and uncontrollable bleeding from the CA are complications that can be encountered during pancreatic surgery. CA repair should be properly managed; otherwise, the patient may experience poor outcomes. Here, we have reported successfully management

\footnotetext{
*Correspondence: sfujioka@jikei.ac.jp

${ }^{\dagger}$ Yuki Takano, Shuichi Fujioka and Hironori Shozaki contributed equally to this article as first authors

${ }^{1}$ Department of Surgery, Jikei University Kashiwa Hospital, 163-1

Kashiwa-shita, Kashiwa, Chiba 277-0004, Japan

Full list of author information is available at the end of the article
}

of CA injury through temporary aortic cross-clamping upstream of the CA during pancreatic surgery in a case.

\section{Case presentation}

A 70-year-old man was referred to our institution by his family doctor for the examination of repeated upper abdominal pain. Preoperative computed tomography $(\mathrm{CT})$ suggested resectable pancreatic cancer of the pancreatic body (Fig. 1A) concomitant with stricture of the CA root (Fig. 1B), which may have been caused by median arcuate ligament (MAL). Pancreaticoduodenectomy with division of the MAL was scheduled. Unexpected bleeding around the CA was observed during surgery, which may have been caused by the injury incurred when the MAL was cut to release CA compression (Fig. 2A, B). As bleeding could be controlled by simple compression only, hemostasis by suturing was attempted first. Contrary to our expectations, the bleeding intensified, making it difficult to confirm the bleeding 


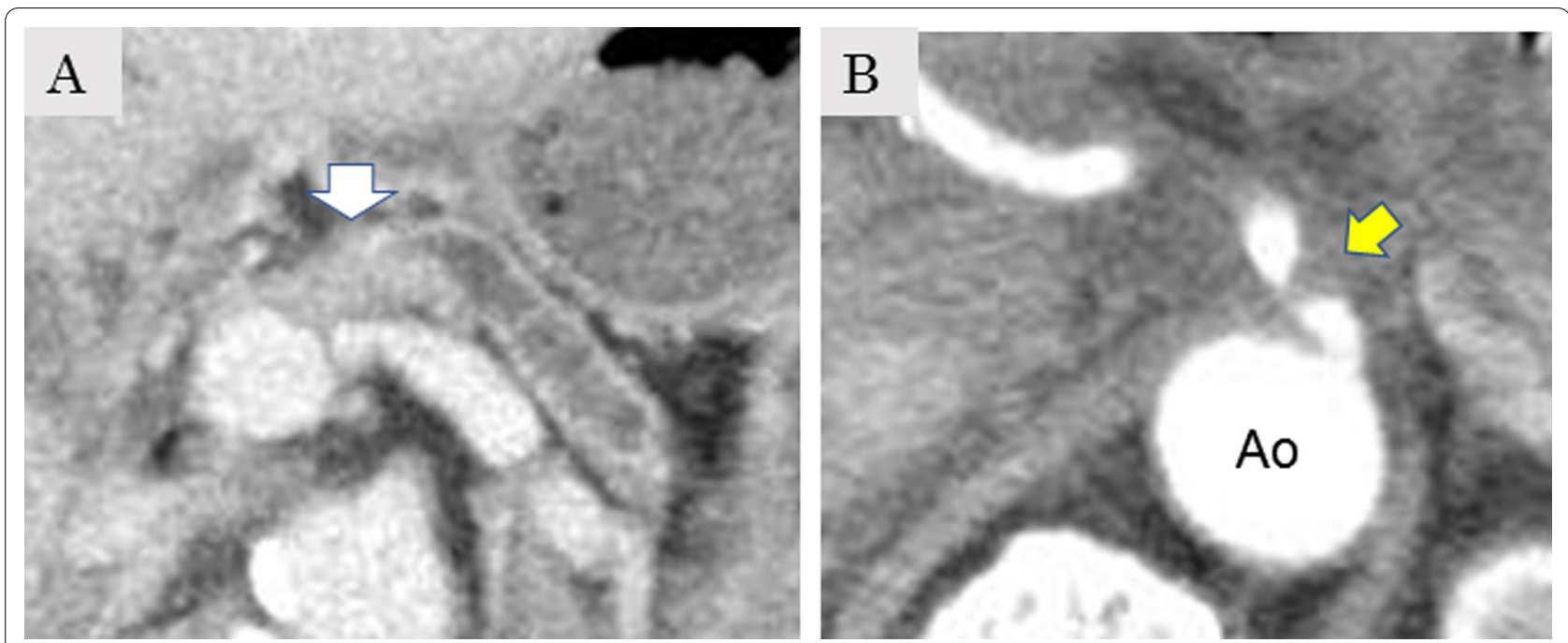

Fig. 1 A Preoperative CT image which represents a resectable pancreatic cancer (white arrow). B Coincidental median arcuate ligament syndrome is shown (yellow arrow)

point. Therefore, we attempted supraceliac aortic crossclamping (SAC) to manage bleeding. To expose the aorta, the crus of the diaphragm was divided, and the aorta was clamped upstream of the CA by a Fogarty vascular-clamp forceps. After performing SAC, the bleeding intensity significantly decreased and a defect of the adventitia measuring $7 \mathrm{~mm}$ in diameter on the CA was confirmed (Fig. 2C). The defect was repaired using a 4-0 Prolene continuous suture (Johnson \& Johnson K.K, NJ, USA). The procedure time for SAC was $2 \mathrm{~min}$ and $51 \mathrm{~s}$, and the intraoperative blood loss was $480 \mathrm{ml}$. The maximum blood pressure increased from 120 to $150 \mathrm{mmHg}$ when SAC was performed and then decrease to $120 \mathrm{mmHg}$ after releasing the clamp. The operative policy was changed to underdo distal pancreatectomy to decrease the risk of hepatic infarction. The patient was discharged uneventfully on postoperative day 19 . A surgical procedure of SAC is shown in Additional file 1.

\section{Discussion}

In our case, the unexpected bleeding may have been due to arteriosclerosis of the CA because fragility at the root of the CA was observed when the MAL was divided. Arteriosclerosis of the CA should be carefully evaluated by preoperative CT, because it can cause up to $60 \%$ stenosis of the CA [4]. In particular, the presence of irregular intraluminal shaggy changes of a certain length is a typical finding that suggests arteriosclerosis of the CA on the contrary compression due to the MAL [5]. In the present case, the stenosis at the root of the CA was diagnosed as compression due to MAL. Median arcuate ligament syndrome (MALS) is the condition which cause clinical presentation associated with direct compression of the celiac artery by the MAL [6]. As the MAL is originally fibrous attachment of the diaphragmatic crura, the treatment of the MALS is dividing the MAL. In most cases with pancreatic cancer, MAL is thought to be diagnosed incidentally by preoperative radiographic examinations, such as dynamic-CT or angiography. These "occult MALS" has considerable significance in performing pancreatic surgery, especially in pancreaticoduodenectomy because of the blockage of the bloodstream to the liver via the gastroduodenal artery. Under the circumstances of CA stricture due to arteriosclerosis, division of the median arcuate ligament has a risk of CA injury. In our case, we attempted SAC, which has been reported as a hemostatic technique for traumatic abdominal pathology [7]. Original procedure of the SAC has been described as exclusion of the aorta by incising the right crus through the lessor sac. This procedure can be easily performed during pancreatic surgery by dividing right crus cranially from the root of the CA. In the present case, the aorta was exposed by dividing the right crus with vessel-sealing device. When the SAC was performed, a defect in the adventitia of the root of the CA was recognized for the first time. This indicates that SAC is useful for temporalizing bleeding from major arterial injuries during pancreatic surgery.

Recently, endovascular vascular occlusion, such as resuscitative endovascular balloon occlusion of the aorta (REBOA), has attracted attention [8, 9]. For aortic injuries below the diaphragm, REBOA is an alternative to resuscitative thoracotomy (RT) with cross-clamping of the aorta to temporalize bleeding prior to cardiovascular collapse. 

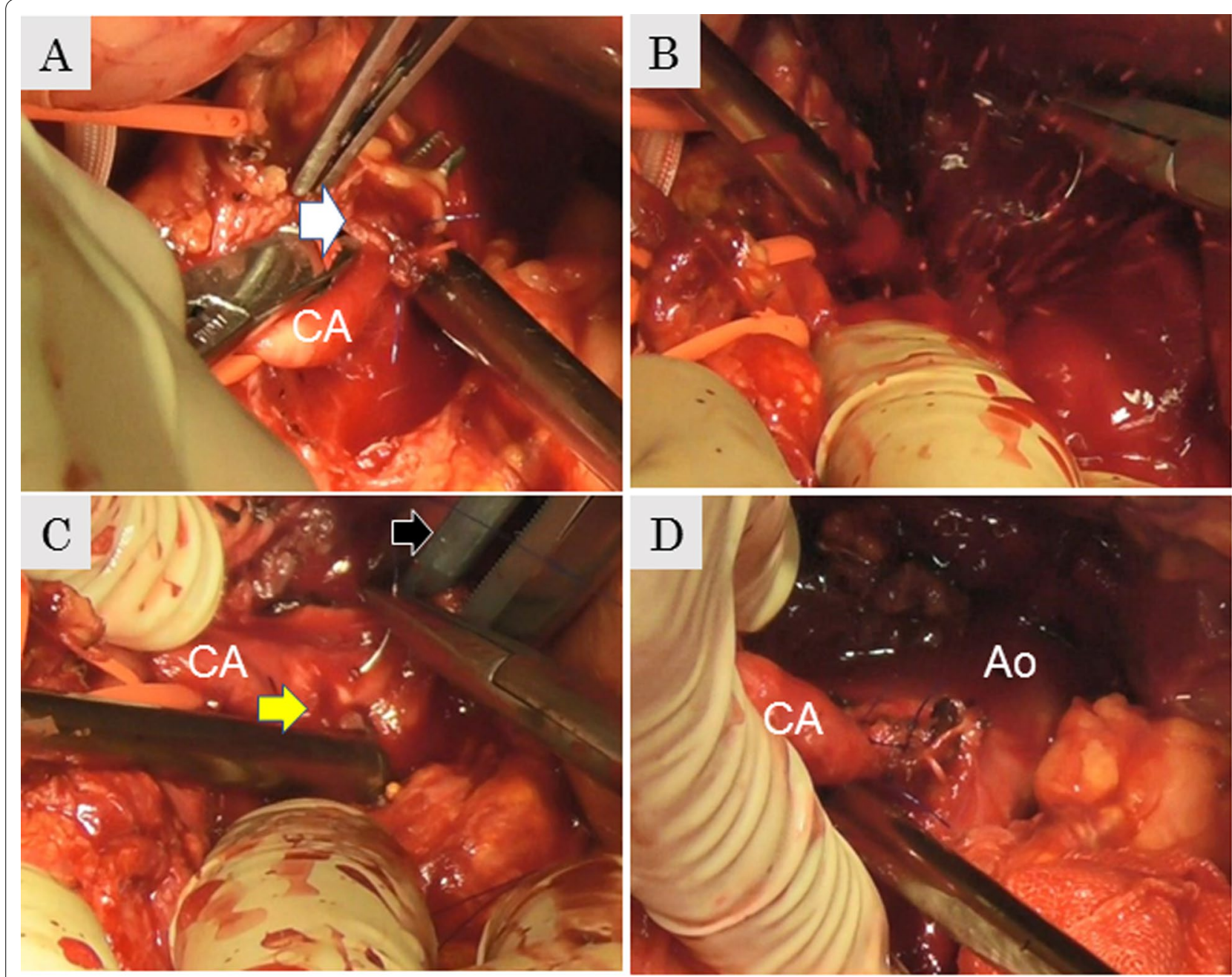

Fig. 2 A Median arcuate ligament (white arrow). B Uncontrollable bleeding from the root of the CA. C A defect on the root of the CA (yellow arrow) recognized after performing supraceliac aortic cross-clamping (black arrow). D After the completion of CA repair, the clamp forceps are released. CA: celiac axis, Ao: aorta

In contrast, REBOA should be performed by an acute care surgeon or interventionalist trained in REBOA and a vascular surgeon for the management of possible vascular complications [10]. Therefore, SAC may be an option between RT and REBOA in the field of abdominal surgery. Easy access to the aorta upstream of the CA is a major advantage of SAC for pancreatic surgery, so as to take the supraceliac aorta immediately. In our case, it took only $3 \mathrm{~min}$ from the initiation of SAC to aortic cross-clamping. Thus, SAC is a useful option for the management of intraoperative arterial injuries during pancreatic surgery.

\section{Conclusion}

Although SAC is primarily a procedure for ruptured abdominal aortic aneurysm, it can be useful for the management of CA injuries during pancreatic surgery.

\section{Abbreviations}

CA: Celiac axis; SAC: Supraceliac aortic cross-clamping; REBOA: Resuscitative endovascular balloon occlusion of the aorta; RT: Resuscitative thoracotomy.

\section{Supplementary Information}

The online version contains supplementary material available at https://doi. org/10.1186/s40792-021-01343-z.

Additional file 1. Intraoperative management for injury of Celiac axis

during pancreatic surgery.

\section{Acknowledgements}

None. 


\section{Authors' contributions}

$Y T, S F, H S$, and NT participated in the treatment of this patients and literature review. TI helped draft the manuscript. All authors read and approved the final manuscript.

\section{Funding}

None.

\section{Availability of data and materials}

Data sharing is not applicable to this article as no datasets were generated or analyzed during the current study.

\section{Declarations}

\section{Consent for publication}

Written informed consent was obtained from the patient for the publication of this case report and accompanying images. A copy of the written consent is available for review by the Editor-in-Chief of this journal.

\section{Competing interests}

The authors declare no competing interests.

\section{Author details}

'Department of Surgery, Jikei University Kashiwa Hospital, 163-1 Kashiwa-shita, Kashiwa, Chiba 277-0004, Japan. ${ }^{2}$ Department of Hepatobiliary-Pancreatic Surgery, Jikei University School of Medicine, Tokyo, Japan.

Received: 10 June 2021 Accepted: 6 December 2021

Published online: 15 December 2021

\section{References}

1. Hirano S, Kondo S, Hara T, Ambo Y, Tanaka E, Shichinohe T, et al. Distal pancreatectomy with en bloc celiac axis resection for locally advanced pancreatic body cancer: long-term results. Ann Surg. 2007;246:46-51.

2. Baumgartner JM, Krasinskas A, Daouadi M, Zureikat A, Marsh W, Lee K, et al. Distal pancreatectomy with en bloc celiac axis resection for locally advanced pancreatic adenocarcinoma following neoadjuvant therapy. Gastrointest Surg. 2012;16:1152-9.

3. Okada K, Kawai M, Tani M, Hirono S, Miyazawa M, Shimizu A, et al. Surgical strategy for patients with pancreatic body/tail carcinoma: who should undergo distal pancreatectomy with en-bloc celiac axis resection? Surgery. 2013;153:365-72.

4. Smith S, Sinclair DM, Satyadas T. Does moderate celiac axis stenosis identified on preoperative multidetector computed tomographic angiography predict an increased risk of complications after pancreaticoduodenectomy for malignant pancreatic tumors? Pancreas. 2007;34(1):80-4.

5. Kim EN, Lamb K, Relles D, Moudgill N, DiMuzio PJ, Eisenberg JA. Median arcuate ligament syndrome-review of this rare disease. JAMA Surg. 2016;151(5):471-7.

6. Goodall R, Langridge B, Onida S, Ellis M, Lane T, Davies AH. Median arcuate ligament syndrome. J Vasc Surg. 2020;71 (6):2170-6.

7. Leschi JP, Coggia M, Goeau-Brissonniere O, Patel JC. Supracoeliac aortic clamping. Technique and indication in traumatic abdominal pathology. Presse Med. 1994;23:1163-5.

8. Qasim Z, Brenner M, Menaker J, Scalea T. Resuscitative endovascular balIoon occlusion of the aorta. Resuscitation. 2015;96:275-9.

9. Biff WL, Fox CJ, Moore EE. The role of REBOA in the control of exsanguinating torso hemorrhage. J Trauma Acute Care Surg. 2015;78(5):1054-8.

10. Brenner M, Bulger EM, Perina DG, Henry S, Kang CS, Rotondo MF, et al. Joint statement from the American College of Surgeons Committee on Trauma (ACS COT) and the American College of Emergency Physicians (ACEP). Trauma Surg Acute Care Open. 2018;3:e000154.

\section{Publisher's Note}

Springer Nature remains neutral with regard to jurisdictional claims in published maps and institutional affiliations.

\section{Submit your manuscript to a SpringerOpen ${ }^{\circ}$ journal and benefit from:}

- Convenient online submission

- Rigorous peer review

- Open access: articles freely available online

- High visibility within the field

- Retaining the copyright to your article

Submit your next manuscript at $\boldsymbol{\nabla}$ springeropen.com 